\title{
Molecular Gerontology: From Homeodynamics to Hormesis
}

\author{
Suresh I. S. Rattan*
}

Laboratory of Cellular Ageing, Department of Molecular Biology and Genetics, Aarhus University, Denmark

\begin{abstract}
The science and study of the biological basis of aging, biogerontology, is now a well-established field with solid scientific base. A paradigm-shift in gerontology has occurred by realising the fact that biological aging occurs in spite of the presence of complex homeodynamic pathways of maintenance, repair and defence, and there is no "enemy within". This viewpoint separates the modulation of aging from the treatment of one or more age-related diseases. A promising strategy in biogerontology is to slow down aging and to extend healthspan by hormetin-mediated hormesis. Physical, nutritional and mental hormetins, which initiate stress responses and strengthen the homeodynamics, are potentially effective aging modulators. As a biomedical issue, the biological process of aging underlies all major diseases, and while the optimal treatment of every disease is a social and moral necessity, preventing the onset of agerelated diseases by intervening in the basic process of aging is the best approach for designing novel pharmaceutical interventions.
\end{abstract}

Keywords: Aging, anti-aging, homeodynamics, hormetics, hormesis, stress.

\section{INTRODUCTION}

Survival of a biological system is a constant struggle between the occurrence of molecular damage and the mechanisms of maintenance and repair [1-3]. There are three major sources of damages to molecules within a cell: (1) reactive chemical species, including free radicals, formed as a consequence of intra-cellular metabolism involving oxygen, metals and other metabolites, and external inducers of damage, such as radiation and pollutants; (2) nutritional components such as glucose and its metabolites; and (3) spontaneous errors in biochemical processes, such as in DNA duplication, transcription, post-transcriptional processing, translation, and posttranslational modifications. Millions of damaging events occur in cells constantly, and in the absence of a wide range of complex molecular, cellular and physiological pathways of maintenance and repair the survival of a system will be impossible. All these processes and their interactions give rise to a network of "homeodynamic space", which is the ultimate determinant of an individual's chance and ability to survive and maintain a healthy state [4, 5]. This review article addresses the questions as to why the survival of an organism is not infinite, why aging occurs, and whether there are effective means of modulating the aging process in order to extend healthspan and longevity.

\section{BIOLOGY OF SURVIVAL AND AGING}

Homeodynamic space is the determinant of an individual's ability to survive and to maintain a healthy state. The traditional conceptual model to describe this property was homeostasis. However, modern understanding of the processes of biological growth, development, maturation, reproduction and aging have led to the realization that the term homeostasis, which means "stability through constancy", fails to incorporate dynamic themes and processes, including interacting networks and complexity. Therefore, the term homeodynamics [6] is being increasingly used to account for the fact that the internal milieu of complex biological systems is not permanently fixed, is not at equilibrium, and is a dynamic regulation and interaction among various levels of organization $[2,5,7]$.

A normal and healthy organism is born with intrinsic, and genetically variable, homeodynamic space, which undergoes expansion during growth, development and maturation [5]. The three

\footnotetext{
*Address correspondenc to this author at the Laboratory of Cellular Ageing, Department of Molecular Biology and Genetics, Aarhus University, Gustav Wieds Vej 10C, Aarhus - C, DK8000 Denmark; Tel: +45 8715 5436;
} Fax:+45 8612 3178; E-mail: rattan@mb.au.dk or sureshrattan@gmail.com main characteristics of the homeodynamic space are: (1) stress response and buffering capacity; (2) damage repair and removal processes; and (3) constant remodeling and adaptation. There are hundreds of genes involved in each of the above components, which comprise the homeodynamic space and its network. However, this protective survival ability is not perfect and absolute due to the complex and interactive nature of biological processes. There is a potential "vulnerability zone" around this protective homeodynamic space, the extent of which can vary among individuals depending on factors such as genetic polymorphism, prenatal exposures, and other growth and developmental conditions. Early life events and life-style factors such as nutrition, infections, mental stimulation and physical activity affect the strength and extent of the homeodynamic space (Fig. 1).

Evolutionary processes have shaped, sharpened and determined the extent and size of the homeodynamic space of a species in order to assure its survival until, at least, reproduction and continuation of generations. This duration of life has been termed as essential lifespan (ELS) [8,9], for which an efficient homeodynamic space is the basic requirement. ELS is generally much shorter than the maximum lifespan potential of an individual with a species or the average lifespan of organisms within the species [10,11]. ELS of a species is determined by genes known as the longevity assurance genes, many of which can be either conserved among species (the so-called public genes) or these can be species-specific (the socalled private genes), depending on the evolutionary life history of the species [3, 12-14].

After the period of ELS, evolution has not selected for any specific gerontogenes with a sole purpose of causing aging and death of the organism. Instead, aging is allowed to happen due to the inefficiency and failure of the homeodynamic processes of maintenance and repair, but without any specific genetic programme [7, 9, $10,15]$. Some other authors, however, have proposed that different kinds of quasi-programme for aging can exist and these may account for eventual senescence and death [16-18]. In any case, one way of conceptualizing aging and senescence is "the progressive shrinkage of the homeodynamic space that happens during the period of survival beyond ELS". During aging, the protective homeodynamic space becomes lesser and lesser with corresponding increase in the zone of vulnerability. This leads to the increased probability of emergence of one or more age-related diseases during aging, and the eventual death (Fig. 1).

The molecular basis for the shrinkage of the homeodynamic space is generally considered to be the accumulation of molecular 


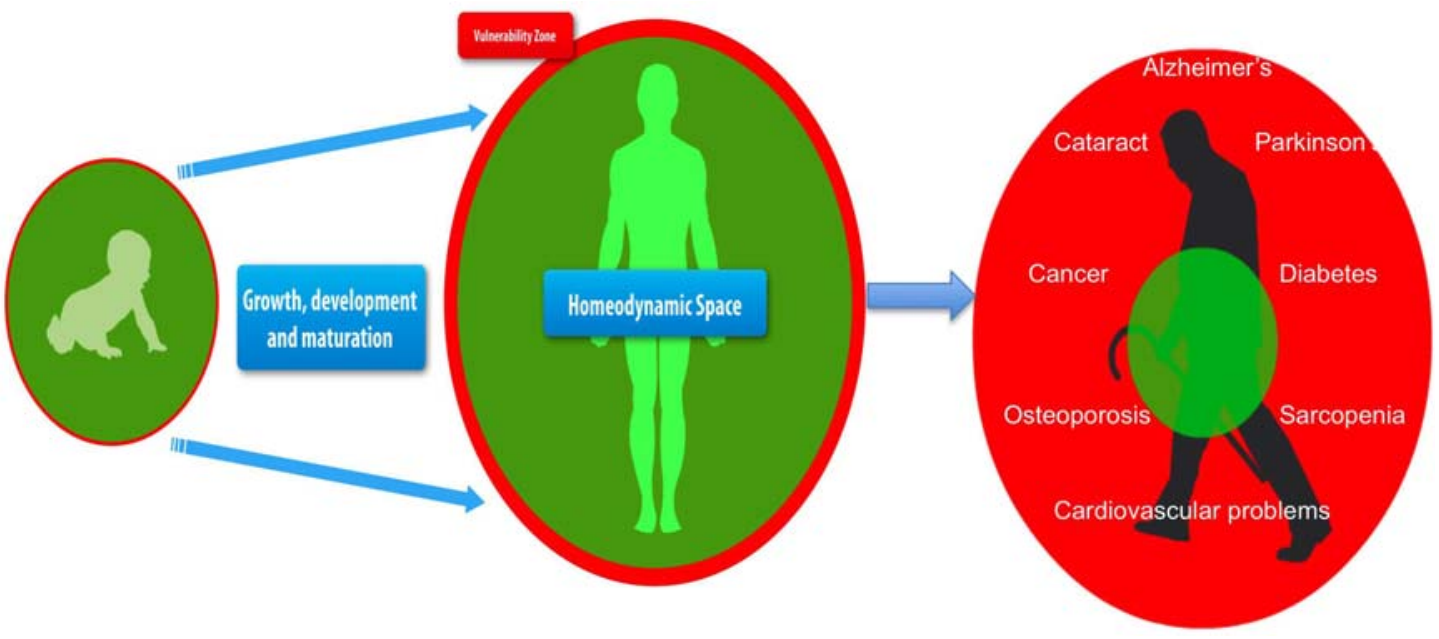

Fig. 1. Homeodynamic space is the ability of the living systems to respond and counteract stress, to repair and remove the damage, and to undergo constant remodelling and adaptation. Due to the imperfections of the maintenance and repair systems, there is always a small vulnerability zone even at a young age, which progressively becomes larger and larger during aging leading to an increase in the chance of appearance of age-related diseases.

damage in all components of the biochemical machinery [2]. Many kinds of damages have been shown to occur in all macromolecules in the cells and in the extracellular matrix and these include DNA damage, RNA base modifications, amino acid misincorporation, non-physiological post-translational modifications, protein misfolding and aggregation, and formation of protein-lipid conjugates such as lipofuscin and advanced glycation endproducts (AGE) [2, 5]. However, such molecular changes vary significantly among different cell types, tissues, organs, system and individuals, and there is a progressive increase in the molecular heterogeneity [2]. At present, it is a challenge to determine the relevance and consequences of each and every kind of molecular damage in functional terms. For example, at what level a particular damage becomes intolerable and causes functional impairments in a particular cell, tissue, organ or individual, and how much such damage must be prevented, repaired or removed for maintaining or regaining function and health?

What is clear, however, that aging at the molecular level occurs due to the progressive failure of maintenance and repair processes leading to a reduced ability to respond, remodel and adapt in the wake of intrinsic and extrinsic challenges. The consequences of a shrunken homeodynamic space are wide ranging. These include altered and disregulated gene expression, genomic instability, epigenetic instability, mutations, epimutations, loss of cell division, cell death, impaired intercellular communication, tissue disorganization, organ dysfunctions, pathology and increased vulnerability to stress and other sources of disturbance.

\section{AGING INTERVENTIONS, STRESS AND HORMESIS}

The piecemeal therapies and replenishment approaches, including stem cells, hormones, antioxidants, vitamins and food supplements, have been shown to have some short-term benefits in acute situations, but have little or no effect on the aging process and its long-term consequences [19-22]. Similarly, gene therapy for aging has serious limitations and only a limited success, mostly in experimental animal model systems, has been demonstrated [14]. More recently, a promising and novel biotechnological strategy for slowing down aging and preventing or delaying the onset of agerelated diseases is that of mild stress-induced hormesis discussed below in the following terms [23-27]:

"The consequences of stress can be both harmful and beneficial depending on the intensity, duration and frequency of the stress. The most important aspect of biological stress response (SR) is that it is not monotonic with respect to the dose of the stressor. SR is almost always characterized by a nonlinear biphasic relationship. Several meta-analyses performed on a large number of papers published in the fields of toxicology, pharmacology, medicine, and radiation biology have led to the conclusion that the most fundamental shape of the dose response is neither threshold nor linear, but is U-shaped or inverted U-shaped, depending on the endpoint being measured".

This phenomenon of biphasic dose response was termed as hormesis [28], and the terminology for hormesis has been further refined to specify the nature of the hormetic responses, such as physiological hormesis, pre-conditioning hormesis, and postexposure conditioning hormesis [29]. More recently, the study and science of hormesis has been termed as hormetics [30].

Hormesis in aging is characterised by "the life-supporting beneficial effects resulting from the cellular responses to single or multiple rounds of mild stress [27]". In the same paper it is mentioned that "the key conceptual features of hormesis are the disruption of homeodynamics, the modest overcompensation, and the reestablishment of homeodynamics. The homeodynamic ability of a biological system is strengthened in a hormetic zone $(\mathrm{H})$ during mild stress, whereas chronic and severe stress results in the progressive weakening of homeodynamics and an increased zone of disruption (D) leading to functional impairments, diseases and eventual death [31]. It is important to note that although the hormetic zone is usually small, both with respect to the dose and the effect, its biological consequences are cumulative, amplified and physiologically significant $[32,33]$.

Hormetins are the conditions or hormetic agents which initially cause slight disruption of homeodynamics, usually by causing some molecular damage, but which then can achieve physiologically beneficial effects by the activation of one or more SR pathways leading to the repair or removal of the damage [30, 34-36]. These hormetins which can strengthen the homeodynamics can be further categorized as:

"(1) Hormetin-P, physical hormetins such as exercise, thermal shock and irradiation; (2) Hormetin-M, mental hormetins, such as mental challenge and focused attention or meditation; and (3) Hormetin-N, nutritional and biological hormetins, such as infections, micronutrients, spices and other natural and synthetic compounds".

It is also mentioned previously that, "an example of stressinduced hormesis is the well-documented beneficial effects of moderate exercise as a hormetin, which initially increases the produc- 
tion of free radicals, acids and aldehydes. Another frequent observation in studies of hormesis is that a single hormetin, such as heat shock (HS) or physical exercise, can strengthen the overall homeodynamics of cells and enhance other abilities, such as tolerance to other stresses, by initiating a cascade of processes resulting in a biological amplification and eventual beneficial effects" [37-40].

Furthermore, it is also said that "various mild stresses that have been reported to delay aging and prolong longevity in a wide variety of cells and animals include temperature shock, irradiation, heavy metals, pro-oxidants, acetaldehyde, alcohols, hypergravity, exercise and food restriction" [27, 41]. Aging modulatory and other effects of hormesis have been reported for human cells in culture. Using a protocol of repeated mild HS to cultured normal human fibroblasts, keratinocytes, endothelial cells, and bone marrow mesenchymal stem cells, several hormetic effects have been reported. These effects include slowing down of cellular aging, extension of cellular replicative lifespan, maintenance of youthful morphology, reduction in molecular damage, and improvement in differentiation, wound healing and angiogenesis [40]. Other hormetins, which have been shown to have aging modulatory effects in human cells, are irradiation, mechanical stretching, and electromagnetic field shock [35].

It has been previously discussed that, "nutritional hormetins, especially those derived from plant sources, have generated much scientific interest for their health beneficial effects. This is because of the realization that not all chemicals found in plants are beneficial for animals in a simple and straight-forward manner. Instead, they initially cause molecular damage by virtue of their electrochemical properties and have a typical biphasic hormetic dose response. Some examples of nutritional hormetins involving heat shock response (HSR) are phenolic acids, polyphenols, flavonoids, ferulic acid, geranylgeranyl, rosmarinic acid, kinetin, zinc, and the extracts of tea, dark chocolate, saffron and spinach [42]".

Discovering novel hormetins by putting potential candidates through a screening process for their ability to induce one or more SR pathways in cells and organisms can be a promising strategy [42]. Recently, a general scheme for screening natural and synthetic single compounds or complex extracts for potentially hormetic use for human beings has been put forward [30]. This scheme is based on the main seven SR pathways within a cell (Fig. 2), which sense and detect the disruption and elicit immediate and delayed SR [31]. Although the exact nature of the initial molecular damage caused by a compound may not be easily identified, activation of one or more SR pathways is a good indicator of the primary action of a potential hormetin.

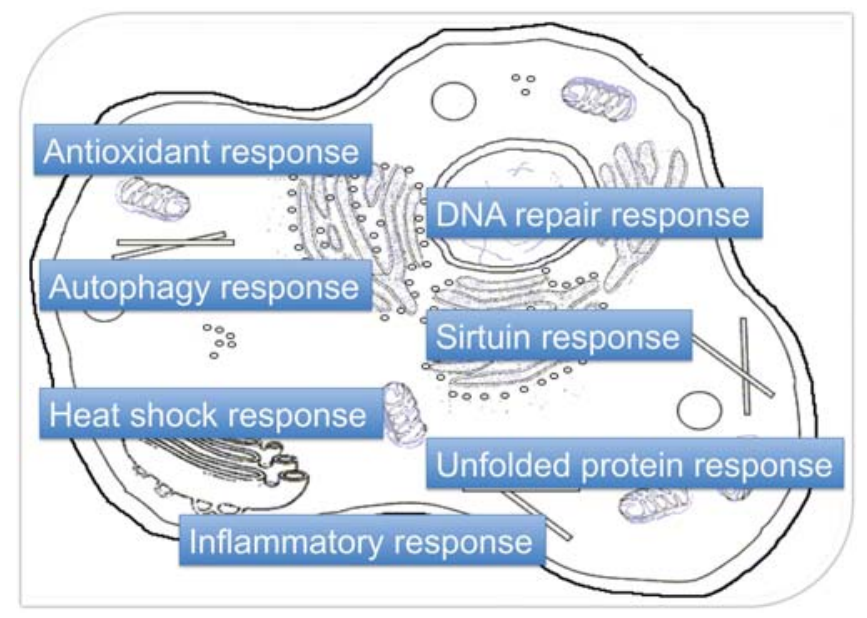

Fig. 2. Major stress response pathways in human cells, which detect disruption of the homeodynamics and initiate a series of maintenance and repair processes.
It is also important to note that "not all pathways of the SR respond to every stressor, and although there may be some overlap, generally SR pathways are quite specific $[31,35,36]$. The specificity of the response is mostly determined by the nature of the damage induced by the stressor and the variety of downstream effectors involved. For example, protein denaturation by heat, heavy metals and antibiotics initiates HSR followed by the activation of proteasome-mediated protein degradation [43, 44]. Similarly, unfolded proteins in the endoplasmic reticulum (ER) induce unfolded protein response (UPR), DNA damage induces the DNA repair response, oxidants induce the Nrf2-mediated antioxidant response, nutritional deprivation induces the autophagy response, energy limitation induces the sirtuin response, and inflammatory agents induce the NF$\mathrm{kB}$ response and initiate the induction of synthesis of a totally different set of proteins and their downstream effectors [45-48].

\section{CONCLUSION}

Since biological aging is now understood as an emergent phenotype due to the failure of homeodynamics and not due to the action of any programmed gerontogenic mechanisms, it has changed our approach towards aging interventions from anti-aging treatment to maintaining health, preserving the homeodynamics, strengthening the homeodynamic space and enhancing the resilience. In that context, a highly promising strategy is that of mild stress-induced hormesis by hormetins-P, $\mathrm{N}$ and $\mathrm{M}$ (physical, nutritional and mental, respectively), which strengthen the homeodynamics and enhance resilience. Applying the concepts from the science of hormetics can also help to discover and develop potentially useful compounds with specific targets for structural and functional maintenance for healthy aging and extended healthspan.

In order to develop novel pharmaceutical interventions for the maintenance of health there is a need to define and establish the immediate and delayed stress response profiles (SRP) during aging. Such SRP should be established at several age-points, which can be the basis for developing the molecular biomarkers of homeodynamic space and defining the health status of cells and organisms. SRP can also be useful for testing potential protectors and stimulators of homeodynamics, and can be a standard for monitoring the efficacy of potential pro-survival, health-promoting and agingmodulating conditions, food components and other compounds.

Often "health" is described either in the context of the absence of one or more diseases or as a vague concept of well-being, without having any objective measures for that. Although some parameters of frailty have been proposed [49-52], measures of health largely remain undefined. Ideally, health can be defined as a state of complete physical and mental independence in activities of daily living (ADL), which perhaps no one has. Therefore, being healthy in realistic terms means having adequate physical and mental independence in ADL at any age, and for which a set of objective measurable parameters at the molecular, cellular and physiological levels are yet to be identified [53]. Understanding the biological basis of aging, combined with the social and psychological aspects of aging, can develop effective and realistic approaches towards maintaining health and extending the healthspan.

\section{CONFLICT OF INTEREST}

The authors confirm that this article content has no conflicts of interest.

\section{ACKNOWLEDGEMENTS}

Laboratory of Cellular Aging (LCA) is financially partially supported by a research grant from LVMH Recherche, France.

\section{DISCLOSURE}

Part of the information included in this article has been previously published in Experimental Gerontology Volume 48, Issue 1, January 2013, Pages 94-98. 


\section{REFERENCES}

[1] Holliday R. Aging is no longer an unsolved problem in biology. Ann NY Acad Sci 2006; 1067: 1-9.

[2] Rattan SIS. Increased molecular damage and heterogeneity as the basis of aging. Biol Chem 2008; 389: 267-72.

[3] Holliday R, Rattan SI. Longevity mutants do not establish any "new science" of ageing. Biogerontology 2010; 11: 507-11.

[4] Rattan SIS. Homeostasis, homeodynamics, and aging. In: Birren J, editor. Encyclopedia of Gerontology. 2nd edition ed. UK: Elsevier Inc.; 2007. p. 696-9.

[5] Rattan SIS. Biogerontology: from here to where? The Lord Cohen Medal Lecture-2011. Biogerontology 2012; 13: 83-91.

[6] Yates FE. Order and complexity in dynamical systems: homeodynamics as a generalized mechanics for biology. Math Comput Model 1994; 19: 49-74.

[7] Rattan SIS. Theories of biological aging: genes, proteins and free radicals. Free Rad Res 2006; 40: 1230-8.

[8] Rattan SIS. Biogerontology: the next step. Ann NY Acad Sci 2000; 908: 282-90.

[9] Carnes BA. What is lifespan regulation and why does it exist? Biogerontology 2011; 12: 367-74.

[10] Carnes BA, Olshansky SJ, Grahn D. Biological evidence for limits to the duration of life. Biogerontology 2003; 4: 31-45.

[11] Olshansky SJ, Biggs S, Achenbaum WA, et al. The global agenda council on the ageing society: policy principles. Global Policy 2011; 2: 97-105.

[12] Martin GM. Modalities of gene action predicted by the classical evolutionary theory of aging. Ann NY Acad Sci 2007; 1100: 14-20.

[13] Holliday R. Genes and the evolution of longevities. Biogerontology 2009; 10: 1-2.

[14] Rattan SIS, Singh R. Gene therapy in aging. Gene Therapy 2009; 16: 3-9.

[15] Rattan SIS. Gerontogenes: real or virtual? FASEB J 1995; 9: 284-6.

[16] Blagosklonny MV. Aging: ROS or TOR. Cell Cycle 2008; 7 : 334454.

[17] Blagosklonny MV. Progeria, rapamycin and normal aging: recent breakthrough. Aging (Albany NY) 2011; 3: 685-91.

[18] Trindade LS, Balduino A, Aigaki T, Heddle JG. Senemorphism: a novel perspective on aging patterns and its implication for dietrelated biology. Biogerontology 2012; 13: 457-66.

[19] Holliday R. The extreme arrogance of anti-aging medicine. Biogerontology 2009; 10: 223-8.

[20] Le Bourg E. Antioxidants and aging in human beings. In: Rattan SIS, editor. Aging Interventions and Therapies. Singapore: World Scientific Publishers 2005; p. 85-107.

[21] Zs-Nagy I. Is consensus in anti-aging medical intervention an elusive expectation or a realistic goal? Arch Gerontol Geriatr 2009; 48: 271-5.

[22] Rizvi SI, Jha R. Strategies for the discovery of anti-aging compounds. Expert Opin Drug Discov 2011; 6: 89-102.

[23] Rattan SIS. Ageing, gerontogenes, and hormesis. Ind J Exp Biol 2000; 38: 1-5.

[24] Rattan SIS. Hormesis in biogerontology. Crit Rev Toxicol 2001; 31: 663-4

[25] Rattan SIS. Applying hormesis in aging research and therapy. Hum Exp Toxicol 2001; 20: 281-5.

[26] Rattan SIS. Mechanisms of hormesis through mild heat stress on human cells. Ann NY Acad Sci 2004; 1019: 554-8.

[27] Rattan SIS. Hormesis in aging. Ageing Res Rev 2008; 7: 63-78.

[28] Southam CM, Ehrlich J. Effects of extracts of western red-cedar heartwood on certain wood-decaying fungi in culture. Phytopathology $1943 ; 33: 517-24$

[29] Calabrese EJ, Bachmann KA, Bailer AJ, et al. Biological stress response terminology: integrating the concepts of adaptive re- sponse and preconditioning stress within a hormetic dose-response framework. Toxicol Appl Pharmacol 2007; 222: 122-8.

[30] Rattan SI. Rationale and methods of discovering hormetins as drugs for healthy ageing. Expert Opin Drug Discov 2012; 7: 43948.

[31] Demirovic D, Rattan SIS. Establishing cellular stress response profiles as biomarkers of homeodynamics, health and hormesis. Exp Gerontol 2013; 48: 94-98.

[32] Calabrese EJ, Iavicoli I, Calabrese V. Hormesis: why it is important to biogerontologists. Biogerontology 2012; 13: 215-35.

[33] Chirumbolo S. Possible role of NF-kB in hormesis during ageing. Biogerontology 2012; 13.

[34] Rattan SIS, Demirovic D. Hormesis and aging. In: Mattson MP, Calabrese E, editors. Hormesis: a revolution in biology, toxicology and medicine. New York: Springer; 2009. p. 153-75.

[35] Rattan SIS, Demirovic D. Hormesis can and does work in humans. Dose Response 2010; 8: 58-63.

[36] Rattan SIS, Demirovic D. Hormesis as a mechanism for the antiaging effects of calorie restriction. In: Everitte AV, Rattan SIS, Le Couteur DG, de Cabo R, editors. Calorie Restriction, Aging and Longevity. Dordrecht: Springer 2010; p. 233-45.

[37] Le Bourg E, Malod K, Massou I. The NF-kappaB-like factor DIF could explain some positive effects of a mild stress on longevity, behavioral aging, and resistance to strong stresses in Drosophila melanogaster. Biogerontology 2012; 13: 445-55.

[38] Candow DG, Forbes SC, Little JP, Cornish SM, Pinkoski C, Chilibeck PD. Effect of nutritional interventions and resistance exercise on aging muscle mass and strength. Biogerontology 2012; 13: 345-58.

[39] Mattson MP, Calabrese E, editors. Hormesis - a revolution in biology, toxicology and medicine. New York: Springer; 2010.

[40] Rattan SIS, Fernandes RA, Demirovic D, Dymek B, Lima CF. Heat stress and hormetin-induced hormesis in human cells: effects on aging, wound healing, angiogenesis and differentiation. Doseresponse 2009; 7: 93-103.

[41] Le Bourg E, Rattan SIS, editors. Mild stress and healthy aging: applying hormesis in aging research and interventions. Dordrecht, The Netherlands.: Springer; 2008.

[42] Rattan SIS, Kryzch V, Schnebert S, Perrier E, Carine Nizard C. Hormesis-based anti-aging products: a case study of a novel cosmetic. Dose Response 2012; in press.

[43] Verbeke P, Fonager J, Clark BFC, Rattan SIS. Heat shock response and ageing: mechanisms and applications. Cell Biol Int 2001; 25: 845-57.

[44] Liberek K, Lewandowska A, Zietkiewicz S. Chaperones in control of protein disaggregation. EMBO J 2008; 27: 328-35.

[45] Banhegyi G, Baumeister P, Benedetti A, et al. Endoplasmic reticulum stress. Ann N Y Acad Sci 2007; 1113: 58-71.

[46] Yoshida H. ER stress and diseases. FEBS J 2007; 274: 630-58.

[47] Basaiawmoit RV, Rattan SIS. Cellular stress and protein misfolding during aging. Methods Mol Biol 2010; 648: 107-17.

[48] Markaki M, Tavernarakis N. The role of autophagy in genetic pathways influencing ageing. Biogerontology 2011; 12: 376-385.

[49] Fulop T, Larbi A, Witkowski JM, et al. Aging, frailty and agerelated diseases. Biogerontology 2010; 11: 547-63.

[50] Hubbard VM, Valdor R, Macian F, Cuervo AM. Selective autophagy in the maintenance of cellular homeostasis in aging organisms. Biogerontology 2012; 13: 21-35

[51] Montesanto A, Lagani V, Martino C, et al. A novel, populationspecific approach to define frailty. Age 2010; 32: 385-95.

[52] Mitnitski, A, Song, X, Rockwood, K. Assessing biological aging: the origin of deficit accumulation. Biogerontology 2013; 14: 709717.

[53] Rattan, S.I.S. Healthy ageing, but what is health? Biogerontology 2013: 14: 673-677. 